DOI: https://doi.org/10.46296/yc.v4i7.0040

\title{
EVALUACIÓN DE TRES RESIDUOS AGROINDUSTRIALES COMO SUSTRATOS PARA CULTIVO DEL PLEUROTUS OSTREATUS VAR. FLORIDA
}

\section{EVALUATION OF THREE AGROINDUSTRIAL RESIDUALS AS SUBSTRATUM FOR CULTIVATION OF THE PLEUROTUS OSTREATUS VAR. FLORIDA}

\author{
Puig-Fernández Yaritza ${ }^{1 *}$; Crespo-Zafra Lourdes Mariana²; Cardona-Soberao Yolexis \\ Roberta $^{3}$; Matos-Mosqueda Luisa ${ }^{4}$; Serrano-Alberni Migdalia ${ }^{5}$ \\ 1, 2, 3, 4 Departamento de Ciencia y Tecnología de los Alimentos de la Universidad de \\ Camagüey Ignacio Agramonte Loynaz (UCIAL), Circunvalación norte km 5 1⁄2, Camagüey, \\ Cuba. \\ ${ }^{5}$ Centro de Estudios de Biotecnología Industrial (CEBI) de la Universidad de Oriente (UO), \\ Ave Patricio Lumumba s/n, Altos de Quintero, Santiago de Cuba, Cuba.
}

*Correo: yaritza.puig@reduc.edu.cu

\begin{abstract}
Resumen
Se presentan resultados del proyecto de investigación "Desarrollo de tecnologías para la elaboración de alimentos a partir de setas comestibles a escala de laboratorio", que se plantea el aprovechamiento de residuos agroindustriales de la localidad como sustrato. El objetivo es la evaluación de la cascarilla de arroz y el afrecho cervecero como sustratos puros para el cultivo del Pleurotus ostreatus, empleando la pulpa de café como referencia. Se utilizó la cepa CCEBI 3024 Pleurotus ostreatus var. Florida. Los parámetros estudiados fueron: eficiencia biológica, rendimiento, diámetro de los cuerpos fructíferos y precocidad. El estudio reveló que el afrecho cervecero no fue un medio propicio para la fructificación de la seta objeto de estudio en las condiciones de producción. El sustrato de referencia pulpa de café superó en cuanto a eficiencia, precocidad y rendimiento a la cascarilla de arroz; sin embargo, este último posee potencialidades para ser empleado en el cultivo de setas comestibles en la localidad de Camagüey por su gran disponibilidad, bajo precio y cercanía de las plantas generadoras, contribuyendo a minimizar su impacto en el medio ambiente.
\end{abstract}

Palabras clave: Pleurotus ostreatus; sustrato, cascarilla de arroz, afrecho cervecero y pulpa de café.

\begin{abstract}
Some results are presented from the research project "Development of technologies for the elaboration of food using edible mushrooms on a laboratory scale", which proposes the use of local agro-industrial waste as substrate. The objective is the evaluation of rice husk and brewers' bran as pure substrates for the cultivation of Pleurotus ostreatus, using coffee pulp as a reference. CCEBI 3024 Pleurotus ostreatus var. Florida was used. The parameters studied were: biological efficiency, yield, diameter of the fruit bodies and precocity. The study revealed that the brewers' bran was not a favorable medium for the fructification of the mushroom under production conditions. The substrate of reference, coffee pulp, surpassed the rice husk in efficiency, precocity and yield; however, this last one has potential to be used in the cultivation of edible mushrooms in the town of Camagüey for its great availability, low price and proximity of the rice mills, contributing to minimize its impact in the environment.
\end{abstract}

Keywords: Pleurotus ostreatus; substrate, rice husk, beer bran and coffee pulp.

Información del manuscrito:

Fecha de recepción: 29 de abril de 2020

Fecha de aceptación: 06 de julio de 2020

Fecha de publicación: 10 de julio de 2020 


\section{Introducción}

La popularidad en la producción de setas comestibles se ha expandido en todo el planeta debido a que esta actividad constituye un emprendimiento alternativo interesante. La ventaja que tiene este cultivo es que se utilizan diversos desechos de las actividades productivas agropecuarias, generalmente de fácil obtención, bajos precios y se obtienen elevados rendimientos en la producción de un alimento sabroso, nutritivo y beneficioso para la salud (Royse, Rhodes, Ohga, y Sanchez, 2016).

Cinco géneros principales comprenden cerca de $85 \%$ de la oferta mundial de setas comestibles. Lentinula edodes (shiitake) es ahora el más ampliamente cultivado, con $22 \%$ de la producción mundial en 2013. Le siguen muy de cerca Pleurotus spp. y Auricularia spp., que cuentan con $19 \quad \%$ y $18 \%$ respectivamente. Agaricus bisporus ocupa el cuarto lugar en términos de producción mundial con $15 \%$ del total. Esta situación demuestra que un cambio sustancial ha ocurrido en cuanto a los géneros que constituyen la oferta mundial de hongos comestibles: hace solo 30 años, $A$. bisporus contabilizaba cerca de 55,8 $\%$ del total (J. Sánchez, Royse, D, 2017).

Por su parte las especies de Pleurotus son las más fáciles y menos costosas para cultivar; muy pocas especies de otros hongos demuestran tal adaptabilidad, agresividad y productividad. Además, constituye una de las setas comestibles más estudiada por su calidad y propiedades medicinales (Cortés, 2016). El alto valor nutricional que posee el Pleurotus ostreatus le ha permitido ser catalogado como la carne vegetal (Cortés, 2016; Martínez, 2017).

Existen múltiples reportes de sustratos y suplementos nutricionales empleados en el crecimiento de Pleurotus sp. Dichos sustratos se pueden clasificar en seis categorías: 1) Pajas (de ajonjolí, arroz, cártamo, cebada, sorgo, trigo, avena y zacate en general); 2) Rastrojos (de maíz, mijo, garbanzo, frijol, etc.; 3) Pulpas (de café, de limón, de cardamomo); 4) Bagazos (de caña de azúcar, citronela, maguey tequilero, henequén, uva, etc.); 5) Residuos forestales (como aserrín, viruta, troncos y ramas); y 6) Otros (como papel, olote, tamo de 
maíz, hojas de piña, fibra de coco, lirio acuático, hojas y tallos de plátano, desechos de la industria textil, etc.) (Piña, 2016). En Cuba se destaca fundamentalmente el uso del bagazo de caña de azúcar, pulpa de café y cáscara de cacao ( $\mathrm{N}$. García, Bermúdez, R., Serrano, M, 2011). Los sustratos utilizados en cada región dependen de los desechos agrícolas disponibles localmente.

La agroindustria del municipio Camagüey posee entre sus principales residuos sólidos los del procesamiento de arroz, el cual origina la cascarilla y como parte de la fabricación de cerveza, el afrecho cervecero. Este último posee gran utilidad en la provincia por sus diferentes aplicaciones, esencialmente como alimento animal. Mientras que la cascarilla de arroz constituye un problema ambiental serio, porque el material es quemado generando emisiones gaseosas que contaminan la atmósfera. En la provincia se ha incrementado el interés en disminuir su impacto ambiental mediante el aprovechamiento integral de este desecho, por lo que representa una alternativa atractiva su utilización en el cultivo de setas comestibles.

En la provincia de Camagüey apenas se reportan experiencias en este tipo de siembra. Solo los resultados de un colectivo de autores que comparó el comportamiento y desarrollo de una cepa de Pleurotus ostreatus a partir de cuatro sustratos orgánicos (residuos) (Benitez, 2013) y ostra a pequeña la propuesta tecnológica para producir biomasa de hongos escala (J. Pineda, 2014). Por su parte, el departamento de Ciencia y Tecnología de los Alimentos de la Universidad de Camagüey lleva a cabo el proyecto "Desarrollo de tecnologías para la elaboración de alimentos a partir de setas comestibles a escala de laboratorio", que se plantea el estudio del Pleurotus ostreatus en sustratos que constituyan residuos agroindustriales de la provincia, por lo que el presente trabajo está encaminado a mostrar los resultados alcanzados en la evaluación de la cascarilla de arroz y el afrecho cervecero como sustratos puros para el cultivo del Pleurotus ostreatus var. Florida, empleando la pulpa de café como referencia. 


\section{Materiales y métodos}

Para la producción de setas comestibles, la unidad experimental (biorreactor) empleada fue la bolsa de polietileno transparente y las respuestas a obtener fueron: observaciones del crecimiento y la fructificación, producción de setas, rendimiento, eficiencia biológica, características de las setas y bioconversión. El diseño experimental se detalla en la tabla 1.

Tabla 1. Diseño experimental para la producción de setas comestibles

\begin{tabular}{|c|c|}
\hline Variables & Niveles \\
\hline Cepa & $\begin{array}{c}\text { Pleurotus } \\
\text { ostreatus var. } \\
\text { florida }\end{array}$ \\
\hline Sustratos & $\begin{array}{c}\text { Pulpa de café } \\
\text { (referencia), } \\
\text { cascarilla de } \\
\text { arroz y afrecho } \\
\text { cervecero }\end{array}$ \\
\hline $\begin{array}{c}\text { Formulaciones } \\
\text { (sustratos) }\end{array}$ & Puros \\
\hline Réplicas & $\begin{array}{c}5 \text { por cada } \\
\text { sustrato y } 3 \text { de } \\
\text { control }\end{array}$ \\
\hline $\begin{array}{c}\text { Masa de } \\
\text { sustrato en el } \\
\text { biorreactor }\end{array}$ & $1 \mathrm{~kg}$ \\
\hline
\end{tabular}

Fuente: Elaboración propia
Se empleó la cepa: Pleurotus ostreatus (florida) CCEBI 3024, pertenecientes a la Colección de Cultivo del Centro de Estudios de Biotecnología Industrial (CEBI) de la Facultad de Ciencias Naturales de la Universidad de Oriente, la cual está adaptada a condiciones tropicales.

Se emplean tres sustratos:

- Pulpa de café de la especie Coffea arábica procedente del Centro de beneficio húmedo "Filé" en el municipio III Frente de Santiago de Cuba, sometida a un proceso de secado solar para su posterior almacenamiento y traslado.

- Cascarilla de arroz, obtenida en la Empresa Agroindustrial de Granos UEB Molino Arrocero Tato Rodríguez Vedo, de la provincia de Camagüey como un subproducto resultante del proceso de obtención del grano de arroz de la especie Oryza sativa. En la fase de pretratamiento este residuo se colocó en un área donde no fuera contaminado por animales $u$ otros factores.

- Afrecho cervecero procedente de la fábrica de cerveza Tínima de la provincia de Camagüey, el cual fue secado al sol para su posterior uso. 


\subsection{Procedimiento empleado para el cultivo del Pleurotus ostreatus}

El cultivo de la cepa Pleurotus ostreatus var. florida sobre los subproductos del café, cebada y arroz, se realizó en condiciones ambientales, utilizando como referencia la metodología de cultivo empleada en el Centro de Estudios de Biotecnología Industrial (CEBI) de la Universidad de Oriente, Cuba. La misma se describe a continuación:

Preparación de la cepa: Estas cepas se encontraban en tubos con extracto agar malta y se resembraron en placas Petri con el mismo medio de cultivo, tomando una pequeña porción del micelio llevándolo al centro de la placa con el asa de siembra, luego se incubó a $28^{\circ} \mathrm{C}$.

Preparación del inóculo: Se utilizaron granos de sorgo, los cuales fueron primeramente remojados en agua por aproximadamente tres días. Seguidamente se colocaron en frascos de vidrio de boca ancha (omnia) y se llenaron a una capacidad de $200 \mathrm{~g}$ de semilla hidratada para luego ser tapados con papel aluminio. Posteriormente los frascos fueron esterilizados (50\% de humedad) en la autoclave durante una hora a $121^{\circ} \mathrm{C}$ y seguidamente se dejaron enfriar a temperatura ambiente en un lugar limpio $y$ cerrado. Una vez enfriadas las semillas en los frascos, éstos se agitaron vigorosamente con la finalidad de separar éstas entre sí y favorecer una aireación e hidratación homogénea. Por último, se inocularon con las cepas y se incubaron a $30{ }^{\circ} \mathrm{C}$ durante 17 días. Al cabo de este tiempo el micelio cubrió todas las semillas y el inóculo estuvo listo.

Preparación de los sustratos: El residuo cascarilla de arroz se hidrató por inmersión en agua de cal $(10 \mathrm{~g}$ de cal por cada litro de agua) durante 24 horas en un tanque de $150 \mathrm{~L}$, para así favorecer la hidratación y ablandamiento del sustrato. Luego se eliminó esta agua e inició el proceso de desinfección, a través de la pasteurización a $90{ }^{\circ} \mathrm{C}$ por una hora, seguidamente se escurrió por 12 horas hasta alcanzar un $54 \%$ de humedad. Los sustratos pulpa de café y afrecho cervecero fueron pasteurizados de igual manera, seguidamente se escurrieron con la ayuda de una malla y se dejaron 
enfriar hasta temperatura ambiente, alcanzando valores de 75 y $74 \%$ de humedad respectivamente.

Etapa de inoculación o siembra: la inoculación que se llevó a cabo de manera homogénea, a razón del 10 $\%$ de la masa húmeda del sustrato. Una vez llenadas las bolsas de polietileno transparentes, estas fueron amarradas con hilo, etiquetadas y ubicadas en estantes dentro del cuarto de colonización. El local en el que se llevó a cabo estas operaciones fue previamente desinfectado y permaneció cerrado para evitar corrientes de aire. El personal estuvo provisto de batas, mascarillas, gorro y guantes estériles.

Etapa de incubación: las paredes y pisos del local de incubación fueron limpiadas y desinfectadas con alcohol y cloro. Como medida adicional se esparció cal por todo el lugar y para la esterilización se utilizó propilenglicol $\left(4 \mathrm{ml}^{\text {por }} \mathrm{m}^{3}\right)$, el cual se dejó hirviendo con ayuda de un mechero hasta que se consumió por completo. La ventana y la puerta existentes se cubrieron con nylon de color negro para lograr condiciones de oscuridad total y evitar el ingreso de los insectos, polvo u otro contaminante físico, además de la colocación de mallas en las mismas.

Al tercer día de incubación, fueron cortadas las puntas de las bolsas para permitir la salida de agua acumulada y al quinto día, se les realizaron perforaciones bien distribuidas sobre toda la superficie con una tijera desinfectada, para permitir un mejor intercambio gaseoso y evitar la acumulación excesiva de humedad. La temperatura se mantuvo de 24 a 25 ○C y la humedad relativa de 80 a 92 $\%$. Los tratamientos se incubaron hasta que el micelio invadió todo o la mayor parte del sustrato de las unidades experimentales.

\section{Etapa de producción o} fructificación: Una vez colonizado el sustrato, el hongo cambió de su fase de crecimiento vegetativa al desarrollo de los cuerpos fructíferos, por lo que se trasladaron las bolsas que presentaban primordios al cuarto de fructificación con las condiciones establecidas para la estimulación y desarrollo de estos.

- Humedad del aire: 90-95\%. Esta se logró mediante el riego de agua de forma manual de 3 a 5 veces en el 
día en paredes y pisos.

También fueron colocadas varias cubetas con agua por toda la habitación.

- Temperatura: $27^{\circ} \mathrm{C}$

- Iluminación: Se logró con lámparas fluorescentes colocadas en el techo y paredes y con la ventana del local abierta.

- No fue necesaria la ventilación forzada del local debido porque las fructificaciones se obtuvieron con pequeños intervalos entre una bolsa y otra. Al ser un número pequeño de biorreactores en dicho espacio, no se produjo un exceso de $\mathrm{CO}^{2}$ en el ambiente.

Etapa de cosecha: La cosecha se realizó de forma manual, cortando los racimos con una cuchilla bien afilada y esterilizada para evitar remover el sustrato ya que los hongos se producen en oleadas, por lo que el sustrato no debe ser maltratado pensando en la próxima cosecha. La segunda oleada se produjo nuevamente a los 7 días, obteniéndose una menor producción.

\subsection{Muestreo y control de la producción del Pleurotus ostreatus}

Para llevar a cabo el muestreo y control de la producción se confeccionó una tabla en la que cada bolsa constituía una muestra, registrándose las observaciones sobre el crecimiento del micelio, las posibles e indeseables apariciones de contaminantes, el tiempo de colonización y de fructificación, el día de aparición de los primordios (precocidad) y de los carpóforos, día de las cosechas producidas y luego con los datos de la producción se calcularon los parámetros de control: eficiencia biológica y rendimiento. Algunas de las variables respuesta que se exponen a continuación:

- Precocidad (P): Definida como el tiempo que transcurre entre el día de la inoculación y el día en que aparecen los primeros primordios 0 carpóforos (N. García, Bermúdez, R., Serrano, M, 2011)

- Producción promedio por bolsas: Definida como la masa total de las setas frescas cosechadas entre el número de bolsas que produjeron (N. García, Bermúdez, R., Serrano, M, 2011) 
- Tamaño de los carpóforos: Esta variable se registra mediante la medición en $\mathrm{cm}$ de los carpóforos cosechados en cada tratamiento (Borja, 2016)

- Características de las setas comestibles. Se evaluaron algunas características de las fructificaciones obtenidas por los aspectos morfológicos: color y textura.

- Masa del hongo fresco: Esta variable se registra masando en gramos la producción de setas por cada tratamiento, esto con la ayuda de una balanza digital (Borja, 2016)

- Eficiencia biológica (EB): Definida como la relación en porciento de la masa de las setas frescas y la masa del sustrato seco expresado en porcentaje (N. García, Bermúdez, R., Serrano, M, 2011).

$$
(E B)=\frac{\text { masa de las setas frescas }}{\text { masa del sustrato seco }} \times 100
$$

- Rendimiento (R): Definido como la relación en porciento entre la masa fresca de las setas y la masa del sustrato húmedo (N. García, Bermúdez, R., Serrano, M, 2011).

$$
(R)=\frac{\text { masa de las setas frescas }}{\text { masa del sustrato húmedo }} \times 100
$$

\section{Resultados y discusión}

\section{Preparación del inóculo}

Para un cultivo eficiente del Pleurotus sp. o de cualquier otro género de setas comestibles, depende en gran medida del mantenimiento y conservación adecuado de las cepas empleadas, siendo el crecimiento del micelio el principal parámetro para evaluar la calidad del inóculo. En la presente investigación la preparación del inóculo fue exitosa, ya que en todos los casos el micelio del hongo creció muy bien en los frascos omnia con los granos de sorgo remojados y esterilizados, observándose en cada uno de los granos un recubrimiento algodonoso blanco, creciendo de forma descendente y pareja hasta lograr cubrir todo el frasco y luego de 17 días, todo el sorgo estaba cubierto por la masa blanca. Este intervalo de tiempo supera la cifra mencionada en la literatura por Guzmán, G. (1993), que apuntó 15 días.

\section{Crecimiento micelial}

El Pleurotus ostreatus colonizó la pulpa de café a los 18 días de inoculación, mientras que, en la cascarilla de arroz, los primordios 
tardaron 7 días más en aparecer, debido a que este es un material difícilmente degradable por el alto contenido de sílice que posee. Los mismos se obtuvieron durante el período antes mencionado, lo que es importante para evitar la contaminación y por consiguiente la inhibición del micelio. Tiempos más prolongados se reporta en bagazo de caña de azúcar con 35 días (Acevedo, 2017). Los primordios se caracterizaron por tener crecimientos protuberantes que permitieron distinguir las copas del respectivo hongo.

Las setas llegaron a su madurez a los cuatro y cinco días después de la aparición de los primordios, indicando que la temperatura no influyó significativamente sobre el tamaño de la oreja (cuerpo fructífero), sin embargo, la cepa de Pleurotus utilizada en el experimento, es un híbrido adaptado a condiciones tropicales, que necesita temperaturas óptimas para su mejor desarrollo durante la etapa de colonización y de fructificación. Se observó que, a temperaturas elevadas, existía pérdidas de calidad y de rendimiento (setas de color más claro, más delgadas y con menos masa).

\section{Desarrollo de los cuerpos fructíferos}

De manera general las setas presentaron un buen desarrollo en ambas fases de crecimiento (incubación y fructificación), observándose que los cuerpos fructíferos crecen en forma de racimos o ramilletes a partir de un tallo o tronco central el cual es corto, apenas de 1-2 cm, estos racimos siempre se orientan en busca de la luz. En las bolsas que presentaban primordios fue necesario realizar perforaciones mayores al nylon para permitir el adecuado brote de las setas y así no dañar la forma y textura de estas. La adecuada iluminación en paredes y techo permitió que los cuerpos fructíferos desarrollaran menos estípite y más píleo (estructura de mejor textura y gusto al paladar).

La cosecha se realizó a los 24 días de la inoculación en pulpa de café y a los 28 en cascarilla de arroz, cuando las orejas estaban completamente planas, con coloración blancuzca o grisácea, y consistencia carnosa típica, como se muestra en la ilustración 1. 
La segunda cosecha se realizó a los 13 días posteriores al primer corte, en una de las bolsas con pulpa de café y en tres de las que contenían cascarilla de arroz, momento en el cual se decidió culminar el experimento. Esto trae consigo un menor valor en la eficiencia biológica y rendimiento final. El proceso de fructificación duró aproximadamente 30 días.

Ilustración 1. Setas listas para su cosecha: a) pulpa de café, b) cascarilla de arroz
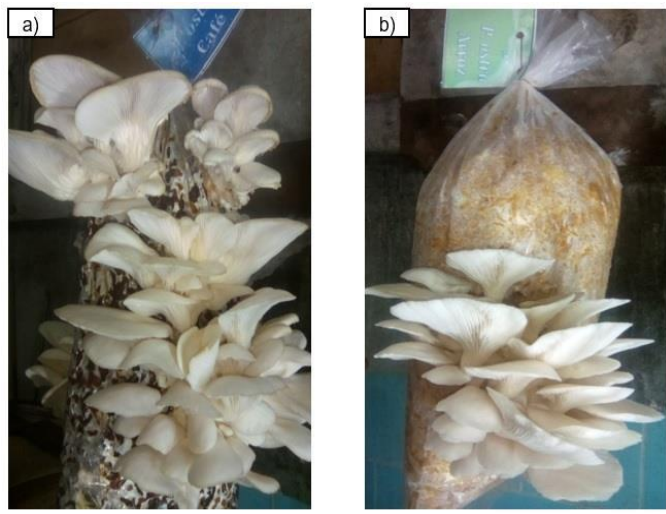

Fuente: Elaboración propia

Pleurotus (florida) en pulpa de café tuvo 138 carpóforos como su mayor cifra, en comparación con la cascarilla de arroz, que solo presentó 120. En cuanto a su apariencia, presentó una coloración blanca y textura carnosa pero frágil con orejas de bordes finos con una ligera ondulación. El mayor diámetro de los cuerpos fructíferos fue de 8 cm en pulpa de café y $9.50 \mathrm{~cm}$ en cascarilla de arroz. Estos resultados coinciden con estudios previos (M. García, 2008).

Resultados de la evaluación de la producción del Pleurotus ostreatus

Pleurotus ostreatus florida obtuvo un valor superior en cuanto a producción total, en pulpa de café con respecto a la cascarilla de arroz, con respectivos valores de eficiencia biológica y rendimiento de 68,02 \% y $17,00 \%$ sobre la pulpa de café y $33,03 \%$ y $15,19 \%$ en cascarilla de arroz. Estos resultados se explican por las diferencias en las características de los dos sustratos utilizados, en cuanto a su composición.

Para que el proceso sea factible económicamente, según establece la tecnología aplicada, los rendimientos deben ser superiores al $10 \%$ y la eficiencia biológica debe alcanzar valores como mínimo del 40 $\%$, por tanto, los resultados de eficiencia biológica y rendimiento obtenidos en la pulpa de café son satisfactorios. En la figura 2 se muestra un gráfico comparativo con los resultados obtenidos por el CEBI en pulpa de café, en el que se muestran valores de eficiencia 
biológica y de rendimiento superiores a los obtenidos en la presente investigación. Esto pudo deberse a que la composición rica en azucares de la pulpa de café, dio lugar durante la etapa de incubación a la aparición de larvas de moscas, las que compitieron con la seta por espacio, oxígeno y nutrientes, influyendo de manera negativa en el adecuado desarrollo del Pleurotus ostreatus en este medio.

Figura 2. Gráfico comparativo con resultados obtenidos por el CEBI en pulpa de café

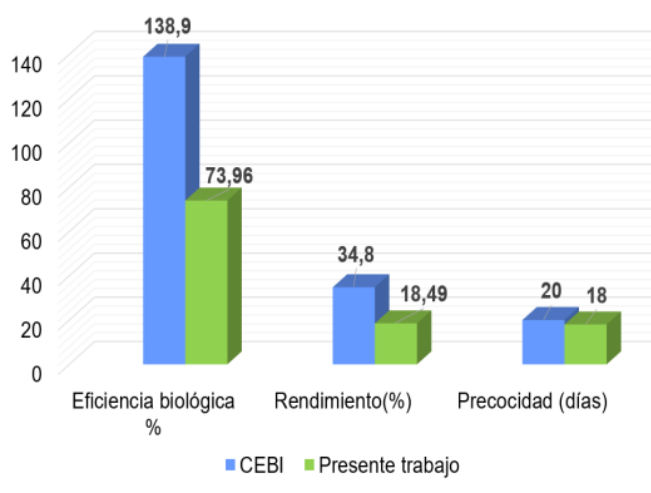

Fuente: Elaboración propia

Resultados alcanzados por (J. Ortiz, 1999) muestran que la cascarilla de arroz pura no posee las mejores cualidades para el cultivo de Pleurotus a escala industrial, debido a que es difícil mantener un $70 \%$ de humedad, requerimiento mínimo establecido para cualquier sustrato. Sin embargo, (Ramón, 2012), obtuvo en $2 \mathrm{~kg}$ de este sustrato y al $10 \%$ de inoculación (200g), una eficiencia biológica de 60,25 \% y un rendimiento de $13,5 \%$ con la misma variedad objeto de estudio.

Por otra parte, las bolsas que contenían afrecho cervecero no presentaron desarrollo por parte del Pleurotus ostreatus. Esto pudo deberse a que el proceso de pasteurización no fuera el adecuado o que este pretratamiento provocara una pérdida de nutrientes. Otra razón pudo ser un alto contenido de partículas finas dando lugar a la compactación del sustrato en las bolsas, lo cual crea pocos espacios intercelulares, se dificulta la aireación tan importante para los hongos y se producen condiciones de anaerobiosis provocando la muerte de este o por la influencia negativa de algún factor físico u operacional (temperatura, humedad, concentración de oxígeno, etc.) que inhibió la fase de crecimiento vegetativa del hongo.

\section{Conclusiones}

Los resultados de la evaluación de la cascarilla de arroz y el afrecho cervecero como sustratos puros para el cultivo del Pleurotus ostreatus var. Florida (cepa certificada CCEBI 3024) arrojaron que, bajo las 
condiciones experimentales empleadas, la cascarilla de arroz es eficaz para el cultivo del Pleurotus ostreatus, pero no es eficiente (bajos indicadores de productividad) y el afrecho cervecero no fue un medio propicio para la fructificación de la seta, mientras que el sustrato de referencia pulpa de café superó a la cascarilla de arroz en cuanto a eficiencia, precocidad y rendimiento.

Se corroboró que la cascarilla de arroz, como sustrato único, no posee las mejores cualidades para el cultivo de Pleurotus debido fundamentalmente a la poca capacidad de retención de agua, por lo que sería conveniente el estudio de mezclas de sustratos que mejoren esta característica.

La cascarilla de arroz constituye un residuo agroindustrial con potencialidades para ser empleada en el cultivo de setas comestibles en la localidad de Camagüey por su gran disponibilidad, bajo precio y cercanía de las plantas generadoras, contribuyendo a minimizar su impacto en el medio ambiente.

\section{Bibliografía}

Acevedo, R. C. (2017). Valoración y crecimiento del cultivo de Pleurotus Ostreatus en cuatro sustratos generados a partir de procesos productivos agropecuarios, en el municipio de Málaga Santander Trabajo de grado, Especialización en procesos de Alimentos y Biomateriales, Universidad Nacional abierta y a distancia UNAD, Málaga.

Benitez, T. P., Fleitas M.D., Batista, L.P., Plana, L.P. (2013). Comparación de substratos para la producción de hongos comestibles. XIV Jornadas de Divulgación TécnicoCientíficas. Jornada Latinoamericana. Facultad de Ciencias Veterinarias Universidad Nacional de Rosario.

Borja, L., Segura, W. (2016). Evaluación de residuos agrícolas predominantes en la provincia Bolívar para la producción de hongos tipo ostra (Pleurotus ostreatus) por fermentación sólida. Trabajo de grado, Ingeniería Agroindustrial, Universidad Estatal de Bolívar, Guaranda, Ecuador.

Cortés S. T. (2016). Calidad microbiológica, físico-química y organoléptica del hongo comestible (Pleurotus ostreatus) fresco y eshidratado, cultivados en tres residuos de cosecha (Tesis de grado), Universidad Técnica Estatal de Quevedo. Ecuador. 
García, M. (2008). Cultivo de setas y trufas (5a. ed.) [en línea]. España: Mundi-Prensa. Recuperado el 26 de febrero de 2018, de http://site.ebrary.com/lib/uasua ysp/Doc?id=10227931\&ppg=1 $\underline{0}$

García, N., Bermúdez, R., Serrano, M. (2011). Formulaciones de sustratos en la producción de setas comestibles Pleurotus. Tecnología Química, 31(3), 1522. Universidad de Oriente, Santiago de Cuba, Cuba.

Guzmán, G. (1993). El cultivo de los hongos comestibles. Instituto Politécnico Nacional. México, D. F.

López, A. (2014). El género Pleurotus y su diversificación taxonómica en especies. Fungicultura, 14.

Martínez Padrón, A. M. (2017). Aprovechamiento de los residuos de la Cáscara de haba (Vicia faba) mediante el cultivo del hongo Pleurotus ostreatus. (Tesis de grado), Escuela Superior Politécnica de Chimborazo. Ecuador.

Ortiz, J. (1999). Contribución al estudio del cultivo de hongos comestibles del género Pleurotus en Cuba. . Tesis de Maestría, Universidad de La Habana, La Habana, Cuba.

Pineda, J. (2014). Desarrollo de una tecnología para la producción a pequeña escala de la biomasa del hongo ostra (Pleurotus ostreatus). Disertación doctoral, Universidad de Camagüey, Camagüey, Cuba.

Piña, A. B., Nieto, D.A., Robles, F. (2016). Utilización de residuos agrícolas y agroindustriales en el cultivo y producción del hongo comestible seta (Pleurotus spp.). Rev. Int. Contam. Ambie., 32, 141-151.

Ramón, P., Ramón, D. (2012). Análisis de la capacidad degradativa de residuos lignocelulósicos utilizando el hongo Pleurotus ostreatus var. Florida. Trabajo de grado, Ingeniería Ambiental, Universidad Politécnica Salesiana, Cuenca, Ecuador.

Royse, D., Rhodes, T., Ohga, S., y Sanchez, J. (2016). Yield, mushroom size and time to production of Pleurotus cornucopiae (oyster mushroom) grown on switch grass substrate spawned and supplemented at various rates. Bioresource Technology, 91(1), 85-91.

Sánchez, J., Royse, D. (2017). La biología, el cultivo y las propiedades nutricionales y medicinales de las setas Pleurotus spp. (1ra ed.). Chiapas, México: El Colegio de la Frontera Sur. 